\title{
Unilateral Reference Values for Hoffmann's Reflex in Patients with Suspected Lumbosacral Radiculopathies
}

\author{
I-Hsuan Tsai*, Hao-Hsuan Tsai \\ Department of Physical Medicine and Rehabilitation, Tri-Service General Hospital, Taipei, Taiwan. \\ Email: *i-hsuan.tsai@uth.tmc.edu, ihtsai137@gmail.com
}

Received May $9^{\text {th }}, 2013$; revised June $9^{\text {th }}, 2013$; accepted June $20^{\text {th }}, 2013$

Copyright (C) 2013 I-Hsuan Tsai, Hao-Hsuan Tsai. This is an open access article distributed under the Creative Commons Attribution License, which permits unrestricted use, distribution, and reproduction in any medium, provided the original work is properly cited.

\begin{abstract}
Unilateral reference values for Hoffmann's amplitude could be applied to the diagnosis of lumbosacral radiculopathy, especially in patients with bilateral lesions. Here, we assessed Hoffmann's reflex by measuring H-wave amplitude and the ratio of $\mathrm{H}$-wave amplitude to $\mathrm{M}$-wave amplitude (the $\mathrm{H} / \mathrm{M}$ ratio). We performed a cross-sectional survey of patients from a Taiwan rehabilitation center $(n=64$, age $20-87)$ who presented with lower back pain that radiated to the leg and received a referral for electrodiagnostic examinations. Reference values for $\mathrm{H}$-wave profile parameters were determined using data from lumbosacral radiculopathy-negative patients $(n=10$, age $22-53)$, who had normal big toe test results, ankle reflex test results, motor and sensory nerve conductive studies and F-wave latency and who displayed no evidence of radiculopathy in electromyography and imaging studies. The $50^{\text {th }}$ percentile values for $\mathrm{H} / \mathrm{M}$ ratio and $\mathrm{H}$-wave amplitude were $28 \%$ and $6.25 \mathrm{mV}$, respectively. An $\mathrm{H}$-wave profile $<5^{\text {th }}$ percentile $(\mathrm{H} / \mathrm{M}$ ratio $<11 \%$ or H-wave amplitude $<2.5 \mathrm{mV}$ ) was significantly related to both electromyography-confirmed current and chronic lumbosacral radiculopathy $(n=64)$. Approximately $41 \%$ of patients with an $H$-wave profile $<5^{\text {th }}$ percentile showed electromyography-confirmed chronic radiculopathy. Electromyography-confirmed current radiculopathy was observed in $35 \%$ of patients with an $\mathrm{H} / \mathrm{R}$ ratio $<11 \%$ and $42 \%$ of patients with an $\mathrm{H}$-wave amplitude $<2.5 \mathrm{mV}$.
\end{abstract}

Keywords: Hoffmann's Reflex; H-Reflex; Electromyography; Electrodiagnostic Studies; Lumbosacral Radiculopathy; Sciatica

\section{Introduction}

The amplitude of Hoffman's reflex (H-reflex) in the soleus-gastronemius complex is a diagnostic criterion with high specificity for patients with lumbosacral radiculopathies [1]. An absent, asymmetric or delayed H-reflex, measured in relation to an individual's height, is considered abnormal [1]. This criterion is difficult to apply in a clinical setting, in which patients vary widely in height and may have bilateral lesions. A unilateral reference for H-reflex amplitude is needed in this context [2].

Accurate clinical diagnosis is challenging in patients with lower back pain that radiates to the leg. Although a subset of these patients suffers from lumbosacral radiculopathy, differentiating between patients with radiculopathy and patients with other contributing conditions may require a clinical or laboratory test. Lower back pain is reported in $49 \%-70 \%$ of the general population, and sciatica is diagnosed in $2.2 \%$ of the general population

"Corresponding author.
[3]. Although any patient with lower back pain that radiates to the leg or positive findings upon physical and neurological examination (e.g., ipsilateral or cross straight leg raising test (SLRT) or Lasègue's sign) may be diagnosed with sciatica, these parameters have poor sensitivity and specificity [3]. Therefore, diagnostic tests are needed to confirm the diagnosis of lumbosacral radiculopathy and differentiate other conditions that may cause similar symptoms, such as lumbar instability, myofascial syndrome or piriformis syndrome [4-7].

For patients with suspected lumbosacral radiculopathy who report no improvement after conservative treatment, electrodiagnostic studies (EDX) or imaging studies are recommended [3]. However, these studies lack sensitivity and specificity for the diagnosis of lumbosacral radiculopathy $[4,8]$. Imaging studies (i.e., magnetic resonance imaging (MRI), computer tomography (CT) and X-ray imaging) are limited when the radiculopathy results from intermittent insult related to posture or chemical irritation $[4,8]$. EDX, including nerve conduc- 
tive studies (NCS) and electromyography (EMG), provide more pathophysiological information, but results vary depending on the chosen diagnostic criteria and the examiner's subjective interpretation [8,9]. NCS results are relatively more objective than EMG results [1]. The H-reflex has been described as the electrophysiologic equivalent of the Achilles muscle stretch (ankle) reflex $[1,9]$. In light of the limitations of current diagnostic tools, the H-reflex has particular value in the diagnosis of lumbosacral radiculopathy [1,9]. The absence of an $\mathrm{H}-$ reflex has high diagnostic specificity for lumbosacral radiculopathy $[1,3]$. When an H-reflex is present, asymmetric amplitude and prolonged latency are considered abnormal $[1,2,10,11]$. However, these criteria are difficult to apply in a clinical setting; the determination of symmetry of the H-reflex is problematic in people with bilateral lesions, and the determination of prolonged latency requires adjustments for the age and height of the patient [8]. Therefore, it is necessary to define a reference for the H-reflex in patients with radiculopathy that can be applied independently of the contralateral side. Previously, only one study defined a unilateral reference for abnormal H-wave amplitude [11]. Moreover, the utility of the ratio of $\mathrm{H}$-wave amplitude to $\mathrm{M}$-wave amplitude $(\mathrm{H} / \mathrm{M}$ ratio) in diagnosing radiculopathy has not been investigated [8]. We designed this study with 2 aims: 1) to define a unilateral reference for H-wave amplitude in individuals with suspected lumbosacral radiculopathy, and 2) to examine the association between unilateral H-wave parameters and chronic or current lumbosacral radiculopathy, as indicated by EMG.

\section{Methods}

\subsection{Data Collection}

This study included patients from a Taiwan rehabilitation center who presented with symptoms suggesting lumbosacral radiculopathy (i.e., lower back pain with or without radiation to the leg, paraesthesia, hypoesthesia or anesthesia in the lumbosacral dermatome, calf or foot muscle wasting, a positive result in the big toe test or a positive SLRT) and received a referral for EDX due to a lack of improvement after at least 2 weeks of conservative treatment (i.e., physical therapy and oral or injectible medication). During the 2012-2013 study period, we collected data from 70 patients, including the results of NCS studies (i.e., tibial motor nerve recording from the abductor hallucis brevis (AHB), sural nerve conduction studies, F-wave latency at the AHB and measurement of the peak-to-peak Mmax-wave (M-wave), peak-to-peak Hmax-wave (H-wave) and $\mathrm{H} / \mathrm{M}$ ratio in the soleus-gastronemius complex) [2,12], EMG studies at two lumbosacral paraspinal muscles and two distal lower limb muscles of the lumbosacral distribution [13], physical examinations (the ankle reflex test and the big toe test), neurological sensory symptoms (i.e., tingling, burning, or stabbing sensations and hypoesthesia or aesthesia) and previous medical and personal histories. Measurement of all parameters was conducted according to the protocol established by Lee (2004) [12] and by a single physician to ensure reliability. Spinal imaging studies were reviewed to check for the existence of lumbosacral root compression, which is an indicator of lumbosacral radiculopathy. After data collection, we excluded 6 people with abnormal sensory NCS that indicated possible peripheral neuropathy. For our final analysis, we included data from 64 participants (64\% male) between the ages of 20 and 87 . None of the included patients had a history of myopathy, mental illness, thyroid disease or a central nervous system deficit, but one patient had a history of transient ischemic attack.

\subsection{Data Analysis}

We performed a descriptive analysis of the study population and a statistical analysis of the collected data. We used the STATA 10 software program for all statistical analyses. A linear regression model with logarithmic transformation was used to examine associations between patient characteristics and $\mathrm{H} / \mathrm{M}$ ratios. Statistics of Kruskal-Wallis and Kendall's Tau examined the association of the characteristics and H-wave amplitude. To determine a reference value for normal $\mathrm{H}$-wave amplitude, we identified a set of lumbosacral radiculopathy-negative patients $(n=10)$ within the study population. The lumbosacral radiculopathy-negative patients had normal results for the big toe test, the ankle reflex test, motor and sensory NCS and F-wave latency; these patients also showed no sign of current (i.e., presence of positive sharp wave or fibrillation (PSW/fi) in any muscle) or chronic (i.e., presence of polyphasic potential $\geq 30 \%$ of all motor unit potentials at $\geq 3$ muscles) radiculopathy by EMG $[1,13]$. The medical histories or image studies of these patients indicated no lumbosacral root compression. We calculated the $5^{\text {th }}, 10^{\text {th }}, 50^{\text {th }}, 90^{\text {th }}$ and $95^{\text {th }}$ percentile for $\mathrm{M}$-wave amplitude, $\mathrm{H}$-wave amplitude and $\mathrm{H} / \mathrm{M}$ ratio for the lumbosacral radiculopathy-negative patients. For subsequent analyses, an abnormal H-wave profile was defined as an H-wave amplitude below the $5^{\text {th }}$ percentile, an $\mathrm{H} / \mathrm{M}$ ratio below the $5^{\text {th }}$ percentile or an H-wave amplitude below the previously established threshold of 1 $\mathrm{mV}$. We then examined the association of abnormal H-wave profiles with current and chronic radiculopathy among patients with normal sensory NCS $(n=64)$ using the Chi-square test with an alpha level of 0.05 .

\subsection{Ethical Considerations}

This study was conducted with the approval and under 
the supervision of the Ethical Review Board of the TriService General Hospital, Taiwan.

\section{Results}

Table 1 shows associations between study population characteristics and measured $\mathrm{H} / \mathrm{M}$ ratios. The study population was $64 \%$ male, with ages between 20 and 87 . Nineteen patients had conditions related to peripheral nerve lesions ( 1 patient had diabetes mellitus, 6 patients had cardiovascular disease, $5^{\text {th }}$ patients had alcoholism and 14 patients were smokers). Seventy-two percent of the patients had symptoms for more than 3 months. Age, sex, ankle reflex test results, current or chronic radiculopathy indicated by EMG, H-wave amplitude, M-wave amplitude and root compression indicated in medical records were the characteristics associated with $\mathrm{H} / \mathrm{M}$ ratios. Sensory symptoms, toe strength and acuity were not related to $\mathrm{H} / \mathrm{M}$ ratios.

Table 2 shows the $5^{\text {th }}, 10^{\text {th }}, 50^{\text {th }}, 90^{\text {th }}$, and $95^{\text {th }}$ percentiles for M-wave amplitude, $\mathrm{H}$-wave amplitude and H/M ratio for patients in the lumbosacral radiculopathy-ne- gative patients. These patients ranged from 22 to 53 years old. The $50^{\text {th }}$ percentile for $\mathrm{H} / \mathrm{M}$ ratio, H-wave amplitude and M-wave amplitude was $28 \%, 6.25 \mathrm{mV}$ and $22.68 \mathrm{mV}$, respectively. The $5^{\text {th }}$ percentile for $\mathrm{H} / \mathrm{M}$ ratio, $\mathrm{H}$-wave amplitude and M-wave amplitude was $11 \%, 2.55$ $\mathrm{mV}$ and $9.20 \mathrm{mV}$, respectively.

Table 3 shows the association between $\mathrm{H}$-wave profile values and EMG-confirmed radiculopathy, as indicated by EMG. We defined an abnormal $\mathrm{H}$-wave profile as an $\mathrm{H} / \mathrm{M}$ ratio $<5^{\text {th }}$ percentile $(\mathrm{H} / \mathrm{M}<11 \%)$ or an $\mathrm{H}$-wave amplitude $<5^{\text {th }}$ percentile (H-wave $<2.5 \mathrm{mV}$ ). We also evaluated the data using the previously established abnormal $\mathrm{H}$-wave amplitude threshold (H-wave $<1 \mathrm{mV}$ ). Approximately $41 \%$ of patients with an abnormal $\mathrm{H}$-wave profile and $54 \%$ of people with an $\mathrm{H}$-wave amplitude $<1 \mathrm{mV}$ were diagnosed with chronic radiculopathy by EMG. Thirty-five percent of people with an $\mathrm{H} / \mathrm{M}$ ratio $<11 \%, 42 \%$ of people with an $\mathrm{H}$-wave amplitude $<2.5 \mathrm{mV}$ and $39 \%$ of people with an $\mathrm{H}$-wave amplitude $<1 \mathrm{mV}$ showed current radiculopathy by EMG. An abnormal H-wave profile was significantly associated with both EMG-confirmed current and chronic radiculopathy.

Table 1. Association of study population characteristics with $\mathrm{H} / \mathrm{M}$ ratio and $\mathrm{H}$-wave amplitude $(\mathrm{n}=64)$.

\begin{tabular}{|c|c|c|c|c|}
\hline \multirow{2}{*}{ Characteristics } & \multicolumn{3}{|r|}{$\mathrm{H} / \mathrm{M}$ ratio } & \multirow{2}{*}{$\frac{\text { H-wave }}{P}$} \\
\hline & $\mathrm{N}$ & $\%$ & $P$ & \\
\hline Sex, men (vs. women) & 41 & $(64.06)$ & $<0.01$ & $<0.01$ \\
\hline Age, mean $(\mathrm{SD})^{\mathrm{e}}$ & 42 & (20) & $<0.01$ & $<0.01$ \\
\hline $\mathrm{PNL}^{\mathrm{a}}$ related conditions & 19 & (29.69) & 0.14 & 0.11 \\
\hline Acuity & & & 0.2 & 0.09 \\
\hline$\geq 6$ month & 41 & $(64.06)$ & & \\
\hline$\geq 3 \&<6$ month & 5 & $(7.81)$ & & \\
\hline$\geq 1 \&<3$ month & 12 & $(18.75)$ & & \\
\hline$<1$ month & 6 & $(9.38)$ & & \\
\hline Negative sensory symptom ${ }^{\mathrm{b}}$ & 28 & $(43.75)$ & 0.7 & 0.69 \\
\hline Positive sensory symptoms ${ }^{\mathrm{b}}$ & 54 & $(84.38)$ & 0.6 & 0.34 \\
\hline Abnormal big toe test & 41 & $(64.06)$ & 0.55 & 0.11 \\
\hline Ankle reflex & & & $<0.01$ & $<0.01$ \\
\hline Absent or + & 25 & $(39.07)$ & & \\
\hline++ & 36 & $(56.25)$ & & \\
\hline+++ or ++++ & 3 & $(4.69)$ & & \\
\hline Current radiculopathy $^{\mathrm{c}}$ & 13 & $(20.3)$ & $<0.01$ & $<0.01$ \\
\hline Chronic radiculopathy ${ }^{\mathrm{c}}$ & 15 & $(23.44)$ & $<0.01$ & $<0.01$ \\
\hline Lumbosacral root compression ${ }^{\mathrm{d}}$ & 26 & $(40.6)$ & 0.01 & 0.01 \\
\hline H-wave $(\mathrm{mV})$, mean $(\mathrm{SD})^{\mathrm{e}}$ & 3.87 & $(3.00)$ & $<0.01$ & \\
\hline M-wave $(\mathrm{mV})$, mean $(\mathrm{SD})^{\mathrm{e}}$ & 19.8 & $(7.34)$ & 0.01 & $<0.01$ \\
\hline $\mathrm{H} / \mathrm{M}$ ratio $(\%)$, mean $(\mathrm{SD})^{\mathrm{e}}$ & 30.1 & $(18.7)$ & & $<0.01$ \\
\hline
\end{tabular}

${ }^{a}$ PNL: peripheral nervous lesions; ${ }^{b}$ Negative sensory symptoms: hypoesthesia or anesthesia; positive sensory symptoms: tingling, burning, or stabbing sensation; ${ }^{\mathrm{c}}$ Confirmed by electromyography; ${ }^{\mathrm{d}}$ Confirmed by spinal images; ${ }^{\mathrm{e}}$ Described with mean and SD. 
Table 2. Percentiles for $\mathbf{H}$-wave profile parameters in lumbosacral radiculopathy-negative patients $(n=10)$.

\begin{tabular}{lccccc}
\hline \multirow{2}{*}{$\begin{array}{l}\text { H-wave profile } \\
\text { parameters }\end{array}$} & $5^{\text {th }}$ & $10^{\text {th }}$ & $50^{\text {th }}$ & $90^{\text {th }}$ & $95^{\text {th }}$ \\
\cline { 2 - 6 } & 9.20 & 13.84 & 22.68 & 28.23 & 28.81 \\
M-wave (mV) & 2.55 & 2.97 & 6.25 & 10.27 & 11.26 \\
H-wave (mV) & 11 & 17 & 28 & 69 & 89 \\
H/M ratio (\%) & & & & & \\
\hline
\end{tabular}

\section{Discussion}

We used a group of lumbosacral radiculopathy-negative patients to define reference values for $\mathrm{H}$-wave profile parameters. The $50^{\text {th }}$ percentile for $\mathrm{H} / \mathrm{M}$ ratio, H-wave amplitude and $\mathrm{M}$-wave amplitude was $28 \%, 6.25 \mathrm{mV}$ and $22.68 \mathrm{mV}$, respectively. We then investigated whether abnormal $\mathrm{H}$-wave profiles were associated with a diagnosis of lumbosacral radiculopathy, as indicated by EMG. Abnormal $\mathrm{H}$-wave profiles, defined as $\mathrm{H}$-wave profiles $<5^{\text {th }}$ percentile (i.e., H-wave amplitude $<2.5 \mathrm{mV}$ and $\mathrm{H} / \mathrm{M}$ ratio $<11 \%$ ) or $\mathrm{H}$-wave amplitude below the previously established reference value of $1 \mathrm{mV}$, were associated with both current and chronic lumbosacral radiculopathy.

Previous studies defined reference values for both $\mathrm{H}$-wave latency and H-wave amplitude. For both parameters, most established reference values are bilateral and pertain to the side-to-side difference. An H-wave latency side-to-side difference $>1 \mathrm{~ms}$ is considered abnormal $[1,10]$. The normal reference value for $\mathrm{H}$-wave amplitude varies among studies. Bilateral $\mathrm{H}$-wave amplitude is expressed as the ratio of the $\mathrm{H}$-wave amplitude of one side to the H-wave amplitude of the contralateral side (the $\mathrm{H} / \mathrm{H}$ ratio). The lower normal limit for the $\mathrm{H} / \mathrm{H}$ ratio ranges from 0.4 to $0.67[2,10,11]$. In the only study to define a unilateral $\mathrm{H}$-wave amplitude reference, Jankus et al. investigated the H-reflex in normal participants and patients with S1 radiculopathy and defined a unilateral $\mathrm{H}$-wave amplitude $<1 \mathrm{mV}$ as abnormal [11]. Ebrahimi et al. $(\mathrm{n}=22)$ reported that the $\mathrm{H} / \mathrm{M}$ ratio is approximately $24 \%$ in healthy patients [14], which is similar to the findings of this study. However, a unilateral H/M ratio reference value for application in the diagnosis of radiculopathy has not been defined. The significance of $\mathrm{H}$-wave amplitude and H/M ratio may be context-dependent. Compound motor action potential decreases when muscles atrophy due to aging, peripheral neuropathy, myopathy or muscle atrophy $[8,9]$. Thus, both the H-reflex amplitude and the M-wave amplitude will decrease upon atrophy of the soleus-gastronemius or the development of a lesion in the efferent pathway of the peripheral nervous system. The M-wave is related to direct activation of all motor neuron pool innervated axons [15], while the H-wave is related to transmission of sig-
Table 3. Associations between $\mathrm{H}$-wave profile parameters and EMG-confirmed lumbosacral radiculopathy $(n=64)$.

\begin{tabular}{|c|c|c|c|c|c|c|}
\hline \multirow{3}{*}{$\begin{array}{c}\text { H-wave profile } \\
\text { parameter }\end{array}$} & \multicolumn{6}{|c|}{ Lumbosacral radiculopathy by $\mathrm{EMG}^{\mathrm{a}}$} \\
\hline & \multicolumn{3}{|c|}{ Current $(\mathrm{n}=13)$} & \multicolumn{3}{|c|}{ Chronic $(\mathrm{n}=15)$} \\
\hline & $\mathrm{n}$ & $\%$ & $P^{\mathrm{b}}$ & $\mathrm{n}$ & $\%$ & $P^{\mathrm{b}}$ \\
\hline $\mathrm{H} / \mathrm{M} \geq 11 \%$ & 5 & 11.9 & 0.02 & 6 & 14.3 & 0.17 \\
\hline $\mathrm{H} / \mathrm{M}<11 \%$ & 8 & 36.4 & & 9 & 40.9 & \\
\hline $\mathrm{H}$-wave $\geq 2.5 \mathrm{mV}$ & 3 & 7.5 & 0.01 & 5 & 12.5 & 0.18 \\
\hline $\mathrm{H}$-wave $<2.5 \mathrm{mV}$ & 10 & 41.7 & & 10 & 41.7 & \\
\hline H-wave $\geq 1 \mathrm{mV}$ & 8 & 15.7 & 0.07 & 8 & 15.7 & 0.04 \\
\hline H-wave $<1 \mathrm{mV}$ & 5 & 38.5 & & 7 & 53.9 & \\
\hline
\end{tabular}

${ }^{\mathrm{a}}$ EMG: electromyography; ${ }^{\mathrm{b}}$ Produced by Chi-square test.

nals between Ia fibers and $\alpha$-motor neurons (mainly slow-twitch motor units) [15]. Therefore, although the $\mathrm{H} / \mathrm{M}$ ratio is generally considered an index representing the level of reflex excitability of the motor pool [1,8], a decreased H/M ratio may instead indicate proximal lesions, such as lesions at Ia afferent fibers proximal to ganglion or conduction blocks at proximal efferent fibers. However, few studies have addressed the application of $\mathrm{H}$-wave amplitude and $\mathrm{H} / \mathrm{M}$ ratio for the diagnosis of radiculopathy [8].

Spinal imaging studies and EDX are recommended for the confirmation of suspected radiculopathies, but limitations exist. In patients whose radiculopathy results from chemical irritations or intermittent insults rather than a static compression, root compression may not be observed in imaging studies [7]. Moreover, reports indicate that MRI studies have a high false positive rate, detecting the presence of spinal root compression in $27 \%$ of patients with no symptoms [9]. EDX provides more physiological information than imaging studies [8]. The presence of $\mathrm{PSW} / \mathrm{fi}$, as indicated by EDX, has $100 \%$ specificity for the diagnosis of radiculopathy, but this finding may fade with time or innervation and is therefore difficult to identify in chronic cases with accumulated root insult and reinnervation $[8,9,16]$. Studies of the morphology of motor unit action potential may provide a diagnosis in chronic radiculopathy cases, but the quantitative method for morphology determination is subjective and time consuming $[1,8,9]$. In the context of these limitations, our finding that low H-wave amplitude and low $\mathrm{H} / \mathrm{M}$ ratios are associated with radiculopathy may provide an objective clinical measurement and a complement for other exams in the diagnosis of both current and chronic radiculopathy [1]. A unilateral H-reflex reference value can also be applied in the diagnosis of patients with suspected bilateral lesions without the need for side-toside measurements [7].

Our criteria for inclusion of patients in the lumbosacral radiculopathy-negative patients were stricter than those 
of previous studies. We surveyed H-wave profile parameters in 10 patients who had normal big toe test results, ankle reflex test results, sensory and motor NCS and F-wave latency and showed no evidence of radiculopathy or root compression in EMG and imaging studies. In contrast, other studies rarely mentioned these objective findings when discussing the selection of normal participants. We studied both $\mathrm{H}$-wave amplitude and $\mathrm{H} / \mathrm{M}$ ratio. We defined an $\mathrm{H}$-wave profile $<5$ th percentile $(\mathrm{H} / \mathrm{M}$ ratio $<11 \%$ or $\mathrm{H}$-wave amplitude $<2.5 \mathrm{mV}$ ) as abnormal. Using these reference values, our study yielded a stricter standard for diagnosing radiculopathy than the previously established $\mathrm{H}$-wave amplitude reference value of $<1 \mathrm{mV}$ [11]. This difference may be due to our use of stricter criteria for the identification of lumbosacral radiculopathy-negative patients. Additionally, we excluded patients with abnormal sensory NCS from our analysis, so the confounding diagnosis of peripheral neuropathy was excluded from our study. There were still some limitations to our study. We determined reference values for H-reflex amplitude in patients with lower back pain that radiated to the leg but normal results upon physical examination; although we suggested that myofascial pain or joint degenerative disease may result in similar symptoms $[5,6]$, these patients may instead have sensory radiculopathy that cannot be detected by laboratory studies [9]. The sample size of our study was small and may be insufficient to establish valid reference values. We assessed $\mathrm{H}$-wave profile parameters with the conventional clinical protocol [12], so the standing and dynamic methods were not investigated [15]. Furthermore, the sensitivity and specificity of our unilateral H-reflex reference values for the diagnosis of lumbosacral radiculopathy were not investigated in this study and need to be determined in the future.

\section{Conclusion}

This study provides unilateral reference values for identifying abnormal $\mathrm{H}$-wave profiles in patients with suspected lumbosacral radiculopathy. An $\mathrm{H}$-wave profile value below the $5^{\text {th }}$ percentile is related to electromyography-confirmed lumbosacral radiculopathy.

\section{REFERENCES}

[1] K. H. Levin, "Approach to the Patient with Suspected Radiculopathy," Neurology Clinics, Vol. 30, No. 2, 2012, pp. 581-604. doi:10.1016/j.ncl.2011.12.011

[2] H. N. Alrowayeh and M. A. Sabbahi, "H-Reflex Amplitude Asymmetry Is an Earlier Sign of Nerve Root Involvement than Latency in Patients with S1 Radiculopathy," BMC Research Notes, Vol. 4, No. 102, 2011. doi:10.1186/1756-0500-4-102
[3] B. W. Koes, M. W. Van Tulder and W. C. Peul, "Diagnosis and Treatment of Sciatica," British Medical Journal, Vol. 334, No. 7607, 2007, pp. 1313-1317. doi:10.1136/bmj.39223.428495.BE

[4] P. Goupille, D. Mulleman and J.-P. Valat, "Radiculopathy Associated with Disc Herniation," Annals of the Rheumatic Disease, Vol. 65, No. 2, 2006, pp. 141-143. doi:10.1136/ard.2005.039669

[5] R. D. Gerwin, "Classification, Epidemiology, and Natural History of Myofascial Pain Syndrome," Current Pain and Headache Reports, Vol. 5, No. 5, 2001, pp. 412-420. doi:10.1007/s11916-001-0052-8

[6] D. S. Binder and D. E. Nampiaparampil, "The Provocative Lumbar Facet Joint," Current Reviews in Musculoskeletal Medicine, Vol., No. 1, 2009, pp. 15-24.

[7] K. P. Barr and M. A. Harrast, "Low Back Pain,” In: R. L. Braddom, Ed., Physical Medicine and Rehabilitation, 4th Edition, Saunders, Philadelphia, 2010, pp. 871-912.

[8] D. C. Preston and B. E. Shapiro, "Electromyography and Neuromuscular Disorders," 2nd Edition, Elseiver, Butterworth Heinemann, Philadephia, 2005.

[9] T. R. Dillingham, "Electrodiagnostic Medicine II: Clinical Evaluation and Findings," In: R. L. Braddom, Ed., Physical Medicine and Rehabilitation, 4th Edition, Saunders, Philadelphia, 2010, pp. 115-222.

[10] T. R. Han, J. H. Kim and N. J. Paik, "A Study on New Diagnostic Criteria of H Reflex," Electromyography and Clinical Neurophysiology, Vol. 37, No. 4, 1997, pp. 241250.

[11] W. R. Jankus, L. R. Robinson and J. W. Little, "Normal Limits of Side-to-Side H-Reflex Amplitude Variability," Archives of Physical Medicine and Rehabilitation, Vol. 75, No. 1, 1994, pp. 3-7.

[12] H. J. Lee and J. A. Delisa, "Manual of Nerve Conduction Study and Surface Anatomy for Needle Electromyography," 4th Edition, Lippncott Williams and Wilkins, Philadelphia, 2004.

[13] H. C. Tong, "Incremental Ability of Needle Electromyography to Detect Radiculopathy in Patients with Radiating Low Back Pain Using Different Diagnostic Criteria," Archives of Physical Medicine and Rehabilitation, Vol. 93, No. 6, 2012, pp. 990-992. doi:10.1016/j.apmr.2012.01.013

[14] H. Ebrahimi and S. Adibeyg, "Evaluation of Posterior Tibial $\mathrm{h} / \mathrm{m}$ Ratio in Cerebrovascular Accidents," Iranian Journal of Neurology, Vol. 7, No. 23, 2013, pp. 266-271.

[15] N. A. Maffiuletti, A. Martin, N. Babault, M. Pensini, B. Lucas and M. Schieppati, "Electrical and Mechanical Hmaxto-Mmax Ratio in Power- and Endurance-Trained Athletes," Journal of Applied Physiology, Vol. 90, No. 1, 2001, pp. 3-9. doi:10.1002/mus.21759

[16] S. C. Cho, M. A. Ferrante, K. H. Levin, R. L. Harmon, Y. T. So and J. Vavricek, "Utility of Electrodiagnostic Testing in Evaluating Patients with Lumbosacral Radiculopathy: An Evidence-Based Review," Muscle and Nerve, Vol. 42, No. 2, 2010, pp. 276-282. 